\title{
sciendo
}

\section{Acting in Order to Know, Knowing in Order to Act: Sosa on Epistemic and Practical Deliberation}

\author{
Jesús Navarro \\ University of Seville \\ DOI: $10.2478 /$ disp-2016-0014 \\ BIBLID [0873-626X (2016) 43; pp. 233-252]
}

\begin{abstract}
The questions 'Do I know p?' and 'shall I take $p$ as a reason to act?' seem to belong to different domains — or so claims Ernest Sosa in his Judgment and Agency (2015), the latest version of his virtue epistemology. According to Sosa, we may formulate the first question in a purely epistemological way — a matter of knowledge "full stop" —, while the second one is necessarily intruded by pragmatic factors - a matter of "actionable knowledge". Both should be answered, in his view, considering the reliability of my belief, but the former could be faced in total abstraction from my personal practical concerns. In this paper I dispute Sosa's view, and claim that no purely epistemic level of knowledge "full stop" is conceivable, at least within a reliabilist framework. A case is put forward in order to show that some given belief may not be considered as reliable by itself, as a token, but always as a member of a type, belonging to some class of reference of other beliefs. And the relevant class of reference may only be chosen considering personal practical interests.
\end{abstract}

\section{Keywords}

Epistemic rationality, practical rationality, virtue epistemology, pragmatic encroachment, agent reliabilism.

The relationship between knowledge and action has become a central issue in many recent debates in analytic epistemology. However, there are many different ways in which knowledge and action may

Disputatio, Vol. VIII, No. 43, November 2016

Received: 19/07/2016 
be related, and those different relations might have been confused at times. I will focus here on two of those relations, which go in opposite directions. First: knowledge may be considered as resulting from some particular kind of action - what is sometimes called a "cognitive performance", but also all of those activities we engage in in order to achieve knowledge. Second: action, or at least some kinds of action, seem to be the result of states of knowledge - in the sense that knowledge is often a reason we adduce in order to justify our doings. Let me call the first sort of relationship AK (action leads to knowledge), and the second one KA (knowledge leads to action). ${ }^{1}$

With respect to the AK relationship, it seems uncontroversial that we only know — at least explicitly and consciously — those contents that we endorse, those beliefs that result from our judgments; since endorsing and judging are things that we do (even if sub-intentionally), knowledge is the result of our doing. Virtue epistemology is an influential trend in analytic philosophy that makes a stronger claim in this respect: not only is knowledge, as a matter of fact, the effect of our cognitive doings: it is constitutively so. If the cognitive state we are in is not the effect of our doing, then virtue epistemologists will deny that it may be considered as knowledge. I.e., an agent who endorses some content $p$ may only be said to know $p$ if his getting it right on $p$ is produced (in non-deviant ways) by her cognitive virtues and faculties. This claim may be spelled out in different ways, for instance: by appealing to the agent's abilities and dispositions they manifest in forming true beliefs, to the credit they earn in the social milieu, to the virtuous character they exhibit, to the appropriateness of her intentions, and so on. All of those views share the target of attempting to account for the distinctive features of knowledge by appealing to the way it is produced by agents. This would allow us to put forward effective solutions to some wellknown epistemological puzzles. For instance, considering knowledge as constituted by the agent's doings would allow us to account

\footnotetext{
1 This is of course related to "directions of fit" (Anscombe 1957; Searle 1983), but the relationship I am discussing here is between knowledge and action, whereas the "direction of fit" is a trait of propositional attitudes, and how they are related to their contents: by adjusting the mind to the world (as beliefs attempt to do) or by proposing ways in which the world ought to adjust to the mind (as desires do).
} 
for situations of knowledge-undermining luck, as the one that affects Gettier cases: an agent who has some justified true belief that is true by sheer luck may not be said to have knowledge, and the reason for this is, according to virtue epistemologists, that the agent has to earn her belief by herself; she has to achieve it. ${ }^{2}$ Virtue epistemology also seems quite promising with respect to the so-called 'value problem'. Plato famously pointed out that states of knowledge do not have more instrumental value than mere true beliefs, at least prima facie. E.g., I will get to the city of Larissa just the same if I know the way to get there and if I merely happen to have the right belief about it — but nevertheless we seem to value states of knowledge over and above those of mere true belief. Why is this so? According to virtue epistemologists, the reason is that we value knowledge because of the way it is produced. Its value stems from the way it is achieved (AK), not from the way it lead us to successful action (KA).

Some other debates in analytic epistemology have been more focussed on the KA relationship. Consider for instance the lively discussion on the "knowledge rule for assertion": we should only assert what we know — or so says Timothy Williamson (1996), who famously claimed that assertion is a kind of action — a speech act - that requires knowledge as its precedent condition in order to be properly performed. Of course, not only assertion, but many other actions seem to have knowledge as its rule. Practitioners, judges, teachers, and virtually every profesional is expected to perform some specific actions only if they know something for sure - and so happens even in our ordinary everyday lives. Paying special attention to the role knowledge plays in the performance of this kind of actions leads philosophers to look at the problem of value in a different sense. From that perspective, the peculiar value of knowledge would be a feature that results from its relevance to practical reasoning, that is, from the role it ought to play in the production of action (KA).

But consider now the possibility of pragmatic encroachment, as it has been defended in epistemology. The question at issue there is a kind of backfire effect from the fact that knowledge may be essentially relevant to understand some kinds of action: the point in this discussion is not just that you may not act unless you have knowledge,

${ }^{2}$ See Sosa 2007, 2015; Greco 2010 and my discussion in Navarro 2015. 
but that you don't have knowledge unless the belief that you have is so good that you could act on it. What is concerning about this idea is that it allows practical interests to intrude epistemic assessments. If Stanley (2005) or Fantl and McGrawth (2007) are right, for instance, our assessment of some cognitive state as knowledge depends on the practical context where the agent intends to act. This position appeared as a radical interpretation of some possibilities raised by epistemic contextualists in the late nineties (Lewis 1996, Cohen 2000). In ordinary situations, contextualists claimed, we may say that an agent knows $p$, even if her evidential basis is relatively poor; but those standards rise when stakes are high. We become more demanding, and so does the agent herself, if she is not epistemically reckless. She should know better, considering what is at stake. Original contextualists had what would later prove to be quite a conservative interpretation of these situations, claiming that epistemic evaluation is distinctive, but context-dependant. However, defendants of pragmatic encroachment would later hold a much more radical view, according to which the change in the practical situation affects the very epistemic assessment of the scene. If this is right, the issue whether an agent knows or not could never be detached from her practical concerns.

I believe that, in a way, the issue of pragmatic encroachment results from the intertwinement of $\mathrm{AK}$ and $\mathrm{KA}$. In order to be virtuous in exercising her epistemic faculties, and consider her own belief as rationally grounded enough to constitute knowledge (which is an AK matter), the agent should consider the context where she intends to perform (which is a KA matter); whereas, at the very same time, in order to properly decide whether to take $p$ as a reason to act or not (KA), she should deliberate on the epistemic strength of her cognitive state (AK). Now, it is hard to deny that this intertwinement introduces a risk of circular reasoning: I will not come to a conclusion in my epistemic reasoning, and be entitled to take my belief as knowledge, unless it is a piece of information so solid than I could act on it; but I will not be able to finish my practical reasoning, and consider my belief as a proper reason for my action, unless it is so good as to constitute knowledge. Imagine an agent holding some justified true belief $p$, where every strictly epistemic feature has been settled. If, assuming pragmatic encroachment, we asked about her: "Does 
she know p?", we would be claiming that the answer to this question is in another question: "Shall she act as if $p$ ?". But, at the very same time, we would be holding that the answer to this second question is in the first one.

If we assumed pragmatic encroachment, then, it seems that we could not solve the AK puzzle until we solve the KA puzzle, while the KA puzzle would send us back to the $\mathrm{AK}$ one, and so on. In the end, if we intended to be strictly rational, we would never be able either to know or to act.

In practice, such kind of circular reasoning is not pernicious because it works like a kind of spiral. The rising of my practical concerns leads me to hesitate about the evidence that I have, forcing me to be more sceptical in my epistemic deliberation, and to search for further evidence that may reinforce my belief up to the point that I consider it firm enough as to constitute both, at the same time, knowledge and a rational basis for action. Fortunately, life does not wait for philosophers to finish their infinite regresses — we would have extinguished long time ago if it did — , but philosophers cannot happily remain in them.

There would be a way out of this vicious circle if we could find a level of epistemic deliberation completely independent of the agent's practical concerns. If such a level were at least conceivable, we could first ascribe this basic sort of knowledge to the agent and then, later (in the logical sense), look at her practical situation in order to decide whether it is convenient for her to act on that piece of knowledge, or if, perhaps, she should know better.

The way I have read it, that is Ernest Sosa's proposal in his Judgment and Agency, when he distinguishes between knowledge "full stop" and "actionable knowledge" (Sosa 2015: 168-91). ${ }^{3}$ We may

\footnotetext{
${ }^{3}$ Sosa is consistent in calling the second kind of knowledge 'actionable', but he has many different labels for the first kind, besides the one of 'full stop' (2015: 178), like those of 'human knowledge' (179), 'what we know period' (179), 'to know something "flat out" ' (180), 'knowledge all right' (187),... I believe all of them have similar senses, at least within the eight chapter of his 2015, which is the main focus of my attention here.
} 
decide, in Sosa's view, whether an agent knows p "full stop" even if we have no idea whatsoever about the practical interests that she had while forming her belief — or even if she had no practical interests at all. The belief deserves to be called 'knowledge', whatever the agent's practical context is, and thus the issue could be settled disregarding the issue whether her belief is good enough as to act on it. ${ }^{4}$

Ernest Sosa's epistemology is a version of agent reliabilism: a view according to which some belief is knowledge if the agent produced it reliably enough. In particular, Sosa holds that the belief must manifest the agent's cognitive faculties and virtues: capacities and abilities that she has, which make her reliable in achieving true beliefs. Doxastic processes, according to Sosa, have the constitutive goal of "getting it right" on some issue. However, when it is a matter of knowledge, we are not only interested in getting it right. The agents' genuinely epistemic deliberations have furthermore as their constitutive goal the one of achieving the truth by themselves, thanks to an exercise of their own abilities (in the sense that the formation of their beliefs would manifest the exercise of their capacities in non-deviant ways). Sosa's theory is then a paradigmatic case of virtue epistemology, as I introduced it in the first section, since it attempts to solve the main problems in the theory of knowledge by teasing out the AK relationship. If an agent's cognitive performance was a real manifestation of the agent's abilities — that is: if she attained the truth "aptly" —, and the agent was "reliable enough" in achieving this, then we may

\footnotetext{
${ }^{4}$ This distinction is not the same as the one between "animal" and "reflective" knowledge that was, and still is, crucial in Sosa's virtue epistemology (Sosa 2007). The animal/reflective distinction has to do with the piece of knowledge being merely reliable ("animal"), or its being achieved by an agent that is aware of her own reliability ("reflective", which in Sosa's views is a matter of second order reliability). Furthermore, Sosa has later defended the importance of defining a third level of knowledge ("knowing full well"), achieved when the belief is not only reliable and the agent is aware of her own reliability, but when the belief is reliable because the agent is aware of her own reliability. There are different ways in which the animal/reflective/full-well trichotomy and the agential/"full-stop" dichotomy may be related, and I am not aware of Sosa being very explicit on this point. The way I interpret him (and I thank Modesto Gómez-Alonso for changing my views on this), the animal/reflective/full-well categorization belongs to the realm of knowledge "full stop", which is a matter of purely epistemic deliberation (see Gómez-Alonso 2014: 25-7).
} 
consider the resulting belief as a piece of knowledge.

But how much is "reliable enough"? Sosa (2015: 172) is aware that that is a highly context-dependant feature. It may not be simply solved as a matter of probability, since each human domain of action sets its own reliability standards. The basketball player may be "reliable enough" if she has a $40 \%$ three-point percentage. A meteorologist may have a considerable ability to predict the weather even if she is short of being $50 \%$ reliable. The domain defines the standard. And, in particular, the standard for epistemic evaluations may also vary depending on practical factors. We may be much more demanding in some situations than in some others, when much is at stake.

Nevertheless, Sosa claims that there is a specific standard for epistemic evaluations, considering achieving the truth as an independent goal of our lives. We may search for truth while performing in many different domains - in so far as any domain's interest ought better be guided by true rather than by false beliefs — but pursuing the truth is, according to Sosa, an action we may consider in isolation from all those practical concerns. Even if we normally do it while trying to get some benefits, it is something we could aim to do on its own. He thus defines a domain of performances, cognitive performances, that has its own rules, its own standards of reliability, its own normative evaluation:

Despite how susceptible we can be to epistemically irrelevant pragmatic factors, there is such a thing as disinterested belief influenced purely by the aim to get it right, to believe correctly (Sosa 2015: 181).

So, the view is that strictly epistemic reliability standards may be defined disregarding practical motivations and the specific domain of practical interest where the resulting belief may be of use. That purely theoretical level of reliability may be assessed, in Sosa's opinion, by considering two requirements: one imposed by memory, and the other by testimony. First, the agent has to consider whether the belief that $p$ she has obtained is safe enough as to deserve to be stored in her memory for later retrieval. And second, the product of her epistemic deliberation must be safe enough as to deserve to be communicated to others. In other words: the goal of epistemic deliberation is to obtain a belief that is good enough to be remembered by the agent herself in the future, and to be communicated to others. 
Memory and testimony would thus allow us to establish a standard for evaluation of our beliefs that is — and even must be - independent of what the agent is doing while she forms her belief. It must be so independent because that same piece of information may later be employed by the agent in the future, with some completely different practical interests, not to speak of those that the recipient of her testimony may have. Given that I, in the future, might not share my current practical aims, and so may happen with others that will perhaps obtain that information from me, my epistemic assessment should abstract from those practical interests.

According to Sosa then, strictly epistemic deliberation settles the standard for knowledge "full stop", disregarding the agent's motivational states (if any), which would be irrelevant for strictly epistemic validity. Those practical interests may have had an important causal role in the belief's aetiology, but they are of no use in order to determine the strictly epistemic validity of the belief.

The reason why memory and testimony are what set the standards for knowledge "full stop" assessments is that, according to Sosa, our epistemic practices are rooted in our constitution as social agents. We are epistemic creatures because we are members of a species that systematically relies on the sharing of information. If information were stored and transferred with extremely poor standards, we would probably be doomed to extinction. And if knowledge were always to be assessed considering the specific practical situation where the agent achieves it, related to the context of her specific practical goals, then our social employment of it would be almost impossible. That is why we store it, so to speak, in abstract, detached from the motivational aetiology that produces it and the practical goals we pursue while forming it, which are not constitutive elements of the resulting epistemic state.

Once this basic level of purely epistemic evaluation is settled, we may later (in the logical sense) want to take the agent's personal practical context into consideration, in order to decide whether that piece of knowledge "full stop" is good enough to be acted on or not. The agent's practical aims, and the risks she assumes in taking her belief as true, become then a crucial factor in her deliberation on what Sosa calls 'actionable knowledge': knowledge the agent may take as a basis for her action. But this level of assessment should be 
distinguished from the purely epistemic one, which is not (or even should not) be affected by those specific practical concerns at all.

Now, this may be read as an attempt to solve the threat of circularity I introduced at the beginning of this paper: the matter whether some given belief is good enough as to be considered knowledge "full stop" would be a purely epistemic issue, independent of any specific practical concerns. In contrast to this, deliberation on whether some given piece of knowledge is good enough as to act on it would be a matter of actionable knowledge, a moment in which considering the specific practical concerns of the agent would be unavoidable. Knowledge "full stop" would thus allow us to isolate a purely AK issue (is the belief correctly produced in order to be called knowledge?), while actionable knowledge would introduce, at a second level, the issue of KA (is my knowledge good enough as to act on it?). The account would be free of circularity because both issues, AK and KA, could be considered serially, being AK independent, and logically previous.

Sosa's strategy to refrain pragmatic encroachment has different moments. The first one is to restrict the effects of pragmatic encroachment to the mixed concept of agential knowledge, which is, as we have seen, half epistemic and half prudential. But he assumes this only under the condition that another purely epistemic level of deliberation be recognised, where such encroachment would be banned:

Thus do we admit a sort of pragmatic encroachment. The relevant difference between the study or seminar room and the market place is constituted by practical concerns. Practical concerns do bear on whether we affirm reliably enough. However, our grade of encroachment need not go all the way to the particular practical context of the believer whose belief is up for epistemic assessment. Social epistemic norms can abstract from such specific contexts (Sosa 2015: 60).

The idea is then that we may abstract from the agent's particular practical context in order to attribute knowledge "full stop" to her. Perhaps her belief would not be reliable enough to act on it in her specific practical situation, but it could be good enough to be 
remembered as known, in general, and to be communicated to others as a piece of knowledge.

But one first problem arises immediately — one Sosa is perfectly alive to: namely that it does not seem possible to consider the belief as worthy or not in total abstraction from practical concerns. Imagine for instance that an agent stored belief $p$ when stakes were very low for her. She simply relied spontaneously on her faculties, and was not considering some possibilities that she should have taken as possible defeaters of her belief under some other high stakes situation. Imagine we clainmed that the belief was good enough to be stored as something known "full stop". Some time later, our agent remembers $p$, and communicates it to somebody else. Is she doing well in making this testimony? That is: should she claim that she knows p? Could that status be properly attributed to her belief, disregarding not only her original situation, when the belief was acquired, but most importantly the specific practical situation where her interlocutor may find herself, while asking for information?

It is hard to make sense of responsibility here, or to spell out the normativity of testimony, unless some idea of practical context is considered. In other words: hardly could knowledge "full stop" be the rule for memory retrieval and testimony if it were absolutely abstracted from its formation process and the level of exigency the agent had while forming it.

But as I said Sosa himself is well aware of this. He does not say that knowledge "full stop" ought to be attributed disregarding practical concerns in general, but only disregarding the agent's practical concern in particular. For that reason, Sosa's virtue epistemology assumes a second form of pragmatic encroachment that is deeper than the one we found in the previous quote, since practical concerns do have a crucial role to play even in the purely epistemic assessment of knowledge "full stop":

The epistemically successful life is a difficult thing to define in general terms, as is the epistemically successful history of a community or species. It seems a matter of collectively attaining and sustaining a picture of the surrounding world that enables a level of prediction, control, and understanding within an acceptable range, given the possibilities and trade-offs proper to the constitution and situation of the subject and/or his group. Here non-epistemic factors do plausibly bear. What deter- 
mines the acceptable range depends on the needs of that life and community, and on the range of possible success allowed by participants' constitution and situation (Sosa 2015: 173, my emphasis).

The particular practical situation of the agent does not affect the strictly epistemic level of knowledge "full stop" deliberation: it only affects the reflection on that piece of knowledge as actionable. Nevertheless, the general practical constitution of agents does have much to say on the basic deliberation on knowledge "full stop". In pure abstraction from this general practical constitution, no deliberation on knowledge (in any sense) would even be conceivable, since it is this general practical realm what defines the set of significant scenarios and possibilities where our beliefs are expected to be reliable enough for later retrieval and testimony.

This move allows Sosa to find a way out of the problem I have just pointed out: even if the agent was in a low stakes situation when formed the belief, she could be responsible while storing it as knowledge "full stop" and, most importantly, when communicating it to others as something known, even if she later is, or her interlocutors are, in a high stakes situations. That is so because the belief was reliable enough considering "the possibilities and trade-offs proper to the constitution and situation of the subject and/or his group".

Therefore, the reflection on whether some belief is good enough to be acted on in general affects the deliberation on whether it is good enough to constitute knowledge "full stop". What distinguishes the deliberation on actionable knowledge is that it is the particular practical situation what ought to be considered. So, in a way, pragmatic encroachment seems to go all the way down to the purely epistemic level of knowledge "full stop". Otherwise, the very idea of reliability would loose its grip.

But Sosa seems to be fine with this. Reflecting on the conditions of reliability for purely epistemic deliberation, we have just seen him claiming that "non-epistemic factors do plausibly bear". That is the reason why he also talks about knowledge "full stop" as "human knowledge": the sort of knowledge that may be expected from us, as human beings. If our biological constitution were completely different, or our practical aims had nothing to do with the ones that we usually have as members of our species, our epistemic standards for 
knowledge "full stop" would doubtlessly vary. That knowledge would not be "human" anymore. Our general biological and practical constitution is inscribed at the very root of our epistemic deliberations.

In that way, Sosa's solution to the AK/KA puzzle would not exactly be to preserve a core of strictly epistemic deliberation for the AK side of the story. Even purely epistemic assessment for Knowledge "full stop" (AK) would in fact be affected by practical factors, only that those would be purely generic: the sort of aims and goals that may be expected from members of our species in general. That level would not be affected by the personal situation of the agent in particular. This would still allow Sosa to solve the risk of circularity, because we would not have to consider the agent's particular practical context in order to deliberate on her knowledge "full stop": we should only consider the species general practical concerns, average limitations and usual capacities. So far, so good — or so it seems.

\section{4}

The conclusion from the preceding section has been that, instead of achieving the ideal goal of isolating strictly epistemic deliberation from all kinds of pragmatic factors, Sosa assumes that pragmatic factors are inscribed in both kinds of epistemic deliberation, only that in importantly different ways: on the one hand, knowledge "full stop" deliberation would be affected by the general practical constitution of the species and group the agent belongs to. Those are an important factor to settle the standard of reliability for knowledge "full stop", and thus to solve the AK puzzle (the exercise of agency that leads to knowledge). On the other hand, deliberation on actionable knowledge would be affected by the specific practical situation of the agent (what is at stake in particular when she intends to act with some practical goal in mind), which would allow her to solve the KA puzzle (the consideration of knowledge as a basis for action). The difference between knowledge "full stop" and actionable knowledge would be that only the latter would be intruded by the specific practical interests of the agent — i.e., her personal goals and stakes.

What I would like to do in this final section is to put forward an objection to this account, based on a case that shows that pragmatic factors must be inscribed in epistemic deliberations at all levels, in an 
even deeper sense - a sense that Sosa would probably reject. If my case works, it shows that the specific practical situation of the agent must also be considered at the level of knowledge "full stop" deliberation, which would otherwise be unworkable. If I am right, not only the general practical constitution of the species or group would affect such deliberation, but also the specific and particular practical situation of the agent.

Sosa believes that the aim of "getting it right", the purely epistemic one of forming a true belief, is what constitutes the proper locus of epistemic assessment. But I will try to show that considering the aim of "getting it right" is not enough: we also have to contemplate the agent's goals, what she intends to do, in order to find out if she gets it right reliably at all.

Here is the case. Let me first introduce the scene deprived of specific practical concerns:

LETTER: Alice has in her hands a disorganized bunch of letters from the 50s, belonging to different authors. Many of those authors suspected at that time that the Government was spying on them, and they were thus prone to introduce false information in their letters. Alice is now reading one of them, written by H.P. Gordon, who is the author of a significant number of the letters in that bunch (but not its majority). In that letter (dated August 10, 1954), Gordon claimed: "I have never met Mr Clark". As a matter of fact, Gordon never suspected he could be spied by anyone, and everything he wrote in his letters was always true, including this claim.

Call $p$ the proposition 'HP Gordon did not meet Mr Clark before August 1954'. Could we say that Alice knows $p$ ? The answer depends, of course, on the reliability of her source. Gordon's letters were reliable, but they were surrounded by those of many other unreliable informants. It was perhaps too easy for her to get similar pieces of information, in the very same way, but to form wrong beliefs inadvertently. We would thus probably hesitate to attribute knowledge to her, since the case is clearly affected by what Pritchard has called 'environmental luck' (2012: 267). Knowledge must be obtained in a reliable way, and if nearby fatal possibilities were too close, we would probably hesitate that what she got was knowledge 
at all, even if she actually got it right. ${ }^{5}$

According to Sosa, we could assess the reliability of that belief disregarding the agent's motivation, if any. We should just consider her cognitive action of forming belief $p$ with the aim of getting it right, on the background of the general practical concerns of our species, and our general constitution, and disregarding what Alice in particular wants to do with that piece of knowledge, and what is at stake for her.

This seems to me quite right with respect to the issue of stakes. Let me exemplify this by imagining two specific practical contexts for LETTER, where only stakes vary:

SERIOUS: If Alice got it wrong, she would loose her job, and her family would die of starvation.

RELAXED: If Alice got it wrong, her idle curiosity would have been satisfied by some wrong belief.

I agree with Sosa that, in this case, we may assess reliability for knowledge "full stop" disregarding what is at stake for the agent, and then later decide on whether that piece of knowledge is actionable for her or not, depending on the agent's specific situation. That is so because the rising of stakes does not particularly affect the set of situations in contrast to which we are considering her judgment (what is called its 'class of reference'). Stakes rising affects how demanding our reliability threshold is, but it does not change anything in the way that reliability is estimated. That is why my objection will not be related to the issue of stakes, but to what we may call the issue of tasks. What I would like to show is that the situation varies hugely if what we change is not the level of risks, but the specific practical task the agent aims to perform.

The fact is that the agent's judgment that $p$ could take place in very different courses of practical action, which would affect the

\footnotetext{
${ }^{5}$ I am aware that nothing in LETTER is said about Alice's awareness of the possibility to find lies in those letters. This would, of course, be crucial for a real situation, but not for what I intend to prove, since it is not a matter of telling animal and reflective knowledge apart (reliability and awareness of one's own reliability). As I will show in a moment, the very assessment of first order reliability is what is at stake, and the possibility that it be affected by practical interests.
} 
relative relevance of nearby possibilities. The specific practical motivations of the agent would make some counterfactual possibilities be much more salient than others, and this would alter the relations of proximity between possible worlds that support the assessment of reliability. This will be clear with the help of two variations of the case, where only the respective practical tasks are different:

FIFTIES: Alice is writing a monograph on ordinary life in the 50s. She is not especially interested in H.P. Gordon's life or opinions in particular, and she may pick and read one of his letters, just as any other one in the bunch.

BIOGRAPHY: Alice is writing H.P. Gordon's biography. She is not particularly interested in any other of the authors of those letters, which she would just skip in order to read only Gordon's ones.

First of all, I would like to highlight the fact that this difference in tasks has nothing to do with a rising of stakes: a FIFTIES context for LETTER may be combined either with SERIOUS or RELAXED versions of the stakes issue. The difference between FIFTIES and BIOGRAPHY has to do with Alice's interests and practical projects, not with what is at stake for her or anybody else. The standards for reliability are not rising in that sense, they could be considered to remain just the same, and to vary depending on what is at stake (SERIOUS vs. RELAXED situations).

My point is that, in order to consider her belief as reliable, we have to decide which are the relevant nearby situations where she could have got it wrong. The belief is not reliable in and by itself, but always considered as a case, a token, belonging to a class of possible beliefs she could have formed - its class of reference. The question is that the difference in practical tasks affects the class of reference that allows us to consider Alice's belief that $p$ as reliable (i.e. as not easily wrong). If Alice were engaged in the task of FIFTIES, she would be reading Gordon's letter just as she could be reading any of the other letters in the collection, most of whose authors were disguising their own opinions in order to elude censorship. We should thus consider the general collection of letters as the set of situations among which only a few — Gordon's letters, a trait that is irrelevant 
for her - would provide Alice with truthful information. She could be reading just any of those letters, but Alice is luckily in front of one whose author is not lying, and what she gets from it is perfectly true: Gordon never met Clark before 1954. However, in that situation she could have got it wrong too easily, a mistake that would have been unnoticed by her. Hardly would we say then that what she got is knowledge, not even knowledge "full stop", since her reliability is extremely low, considering the whole bunch of letters as the class of reference.

But imagine now Alice being engaged in the task of BIOGRAPHY. She would not be interested at all in those letters in her collection that belonged to the other authors, which she would skip in order only to read those written by Gordon. In that case, the relevant class of reference is not the whole bunch of letters, because she would have a strong attentional bias towards those belonging to Gordon. It would be very rare her to stop and loose her time reading a letter from somebody she is not interested at all in — and that is why those other letters ought not be considered as members of the class of reference that we have to take into consideration in order to assess her reliability. Now, in BIOGR APHY, Alice would be obtaining a true belief (Gordon never met Clark before 1954) from a source (letters of Gordon) where most information is truthful. The possibility that Alice could have got some wrong information would not be modally nearby, even if it would be spatially nearby: the letters from other authors who contain a bunch of lies are there, perhaps even adjacent to the one she is reading, but they would just be ignored by her, not because she knows they contain many lies, but because she is not (practically) interested in then, given that she is involved in the task of BIOGR APHY.

So, I would agree with Sosa that considerations on what is at stake should not affect our deliberation on knowledge "full stop". It is just the general practical constitution of human beings what ought be considered in that respect, and not the specific risks the agent is facing. The point where I disagree with Sosa is the claim that the specific practical task the agent may be involved in is also a dismissible feature of the scene, defined by her goals and interests. Rather, I believe that we must take those factors into consideration even for strictly epistemic deliberation, since otherwise we would 
have no reason to choose one set of possibilities as the relevant one among other alternative sets. The interests of the agent are at least an important factor in order to define the relevant class of reference against which we may consider her belief formation process. In abstraction from such practical concerns, our decision about how to meassure her reliability would be simply arbitrary. There is no reason to decide whether she is reading a letter among many that contain lies (the whole bunch), or one among some that only contain truths (those written by Gordon). Spatial proximity ought not be assumed as the only relevant feature to evaluate modal proximity. In the case of BIOGRAPHY, spatially faraway letters would be modally much closer than those that may be spatially adjacent to the one she is reading. The possibility that she could have read a different letter by Gordon is much more relevant in that case than the possibility that she could have read a letter by some of the other authors, given her particular practical interests. ${ }^{6}$

In contrast to the issue of stakes, the one of tasks does not just alter the standards of demand for reliability, but the class of reference against which that reliability is meassured. The interest bias would show that not all counterfactual possibilities supporting our reliability assessments are equally important. If we tried to abstract Alice's affirmation (her attempt to "get it right") from those practical aims, the issue of reliability could not be solved but in an arbitrary way? ${ }^{7}$.

${ }^{6}$ Another way of making my claim here is by saying that we should not take for granted that "environment" (in Pritchard's notion of "environmental luck"), ought be understood in merely spatial and temporal terms. It would be more wise to define it in relation to the possible courses of action the agent may be engaged in, regarding her goals and interests, and the practical plans they give rise to.

${ }^{7}$ The issue I am raising here is a version of the generality problem (i.e., the fact that we have to decide on the set of contexts where the agent may be considered as reliable). Let me recall this problem in a nutshell (I follow here Greco 2007: 59): as we have seen, reliabilism is the view that only beliefs produced by reliable cognitive processes may be considered epistemically justified. The generality problem is an effect of the fact that justification attaches to belief tokens, whereas reliability attaches to process types, and any belief token may fall under many process types. Therefore, we have to decide which type is the one implemented by the token before we find out whether it is reliable or not. This problem was originally proposed as a devastating objection to reliabilist theories of justification - although it may be extended to other theories, as Bishop (2010) has 
Getting it right on the issue whether $p$ is something she may be doing quite reliably in BIOGRAPHY, but not reliably at all in FIFTIES. Does Alice know that $p$ then? That depends on the specific practical task she is facing. Whether or not what an agent believes may count as knowledge is an issue that strictly depends on what she is aiming at.

To summarise, the case here proposed would have shown that, within a reliabilist framework, as is the one proposed by Sosa, no level of epistemic deliberation may be defined that is completely independent from the agent's specific practical concerns. It might be objected that this is quite an ambitious claim, which could hardly be proved by just one single imagined case. And that is perfectly right: if this case proves anything at all, it is because it instantiates a pattern that may potentially affect all epistemic deliberations — as happened with Gettier cases, whose relevance did not rely on Edmund Gettier's excogitation of half a dozen situations. What the general pattern shows is that modal proximity is an issue that we should approach taking the specific practical interests of the agent into consideration - and an infinite number of cases may be envisaged employing this same idea ${ }^{8}$. If we

convincingly shown. I don't find it devastating, but just an interesting question we must have an answer to, if we want to develop the reliabilist framework. The original version of the problem was mostly related to the issue of defining the relevant faculties that intervened in the belief formation process. Somebody's perceptual belief, for instance, could be considered to be produced by perception, visual perception, visual perception in broad daylight, etc., which are process types that vary in their degree of reliability. The challenge for reliabilism is to specify which level of generality is the appropriate one for purposes of evaluating the belief token in question. My variation of this argument applies it to practical tasks: we have to find out whether Alice's belief that $p$ results from her attempt to write a monograph on the FIFTIES, or from her attempt to write Gordon's BIOGR APHY, or just from her idle curiosity, and the resulting reliability would vary depending on the different attentional biases those activities would imply. A similar employment of the generality problem was made by Stitch (1990), in order to argue for a culture-dependant account of the concept of knowledge.

${ }^{8}$ For instance, it could be alleged that Goldman's (1976) famous fake barns case is only based on the presence of barn-looking objects in Henry's surround- 
make sense of reliability in terms of modal proximity (in the sense that the agent is reliable if she would succeed in most nearby possible worlds), then we are in need of a criterion to choose the relevant set of possible worlds. In order to do so, spatial or temporal proximity is certainly not the only feature to consider, and probably not even the most important one. On the contrary, the agent's interests and plans seem to make a crucial difference in order to grasp the possible set of situations that should be considered as the relevant class of reference for our reliability assessments. That would explain why, pace Sosa, not only the general constitution of the species must be considered in order to define a putative level of knowledge "full stop", but also the specific practical goals the agent is pursuing while forming her belief.

The way I see it, there is a strong pragmatist moral to be followed from this argument - although I may just have given here some reasons in its favour that are far from being conclusive. Namely: that beliefs are, constitutively, instruments, and not pictures or static representations. The epistemic quest makes sense because it is a part of action, not because it is an action apart that may be theoretically reconstructed as a matter of pure static contemplation. This is the strongly pragmatist thesis I would endorse, in contrast to Sosa's attempt to contain pragmatic encroachment by defining a separate realm for purely epistemic deliberation, isolated from practical concerns. ${ }^{9}$

Jesús Navarro Departamento de Metafísica y Corrientes Actuales de la Filosofía

ings. But if Henry were pursuing some practical task regarding nearby barns, his attention and interests could be biased in crucial ways which would introduced other modally relevant factors in the situation, besides mere spatial proximity.

${ }^{9}$ Thanks to the audiences of the Network on Epistemology and Society (EpiSoc) meeting "Una jornada con Ernest Sosa," held in the Universidad Carlos III de Madrid in March 2015, and the "I Blasco Disputatio: Pragmatism" conference, held in the Universidad de Valencia in October 2015, for helpful feedback on the material of this paper. Thanks also to Teresa Bejarano and J. Adam Carter for insightful commenting on previous versions of the paper, and to Modesto GómezAlonso, Josep Corbí and the Epistemology Reading Group of the Eidyn Research Centre in The University of Edinburgh for valuable discussion. Research for this paper was supported by the MINECO (Ministerio de Economía y Competitividad) via research Grants FFI2014-51811-P and FFI2015-67569-C2-1-P, and the V Plan Propio de Investigación of the Universidad de Sevilla. 
Universidad de Sevilla Calle Camilo José Cela, s/n 41018, Seville, Spain jnr@us.es

\section{References}

Anscombe, G.E.M. 1957. Intention. Oxford: Blackwell.

Bishop, Michael A. 2010. Why the generality problem is everybody's problem. Philosophical Studies 151(2): 285-98.

Cohen, Stewart. 2000. Contextualism and skepticism. Nous 34(1): 94-107.

Engel, Pascal. 2009. Pragmatic encroachment and epistemic value. In Epistemic Value, edited by Adrian Haddock, Alan Millar, and Duncan Pritchard, Oxford University Press: 183-203.

Fantl, J. and McGrath, M. 2007. On pragmatic encroachment in epistemology. Philosophy and Phenomenological Research 75(3): 558-589.

Greco, J. 2007. The nature of ability and the purpose of knowledge. Philosophical Issues 17(1): 57-69.

Greco, J. 2010. Achieving knowledge. Cambridge: Cambridge University Press.

Goldman, A. I. 1976. Discrimination and perceptual knowledge. Journal of Philosophy 73: 771-91.

Gómez-Alonso, M. 2014. Introducción. In Con Pleno Conocimiento by E. Sosa, translated by M. Gómez-Alonso. Zaragoza: Prensas Universitarias de Zaragoza, 2014.

Lewis, David K. 1996. Elusive knowledge. Australasian Journal of Philosophy 74: 549-67.

Navarro, Jesús. 2015. No achievement beyond intention: a new defence of robust virtue epistemology. Synthese 192(10): 3339-69.

Pritchard, Duncan. 2012. Anti-luck virtue epistemology. Journal of Philosophy 109: 247-79.

Searle, John R. 1983. Intentionality. An Essay in the Philosophy of Mind. Cambridge: Cambridge University Press.

Sosa, Ernest. 2007. A Virtue Epistemology: Apt Belief and Reflective Knowledge 1. Oxford: Clarendon Press.

Sosa, Ernest. 2011. Knowing Full Well. Princeton: Princeton University Press. Sosa, Ernest. 2015. Judgment and Agency. Oxford: Oxford University Press.

Stanley, Jason. 2005. Knowledge and Practical Interests. Oxford: Oxford University Press.

Stich, Stephen P. 1990. The Fragmentation of Reason: Preface to a Pragmatic Theory of Cognitive Evaluation. Cambridge, Mass: MIT Press.

Williamson, Timothy. 1996. Knowing and asserting. The Philosophical Review 105(4): 489-523. 